\title{
REPAIR PROCESS OF SURGICAL DEFECTS FILLED WITH AUTOGENOUS BONE GRAFTS IN TIBIAE OF DIABETIC RATS
}

\author{
Jônatas Caldeira ESTEVES ${ }^{1}$, Alessandra Marcondes ARANEGA², Albanir Gabriel BORRASCA \\ Cristiane Mara Ruiz de Souza FATTAH², Idelmo Rangel GARCIA-JÚNIOR ${ }^{2}$
}

1- DDS, Department of Surgery and Integrate Clinic, Dental School of Araçatuba, State University of São Paulo. Araçatuba, SP, Brazil.

2- DDS, MSc, PhD, Assistant Professor, Department of Surgery and Integrate Clinic, Dental School of Araçatuba, State University of São Paulo. Araçatuba, SP, Brazil.

Corresponding address: Dr. Jônatas Caldeira Esteves - Rua João Gonçalves do Nascimento, 1425, Jardim América - 15570-000 Cardoso, SP, Brasil - Phones: +55-17-3453-1134 ou +55-17-9603-0305. e-mail: jonatasce@hotmail.com

Received: January 9, 2008 - Modification: March 12, 2008 - Accepted: April 18, 2008

\begin{abstract}
F Crom tissue healing is affected under diabetic conditions, major changes might take place in the revascularization, incorporation, replacement and remodeling phases of the grafted area. The purpose of this study was to assess the bone healing process in surgical wounds prepared in tibiae of diabetic rats and filled with autogenous bone. Forty male rats (Rattus norvegicus albinus, Wistar) were randomly assigned to receive an endovenous injection (penile vein) of either citrate buffer solution (Group 1 - control; $\mathrm{n}=20$ ) or streptozotocin dissolved in citrate buffer solution (35 mg/kg) to induce diabetes (Group 2 - diabetic; $\mathrm{n}=20$ ). After determination of glycemia, the animals were anesthetized and the anterolateral regions of the tibiae of both limbs were shaved, antisepsis was performed and longitudinal incisions were made in each limb. The tibiae were exposed and two 2mm-diameter surgical cavities were prepared: one in the right limb, filled with particulate autogenous bone and the other in the left limb, filled with blood clot. The animals were euthanized at 10 and 30 postoperative days. The anatomic pieces were obtained, submitted to laboratory processing and sections were stained by hematoxylin and eosin and Masson's Trichrome for histomorphologic and histometric analyses. In both groups, the wounds filled with autogenous bone graft showed better results than those filled with blood clot. The control group showed higher new bone formation in wounds filled with autogenous bone graft at 30 days than the diabetic group, but without statistical significance. It may be concluded that, in general, the new bone formation occurred with autogenous graft was quantitatively similar between control and diabetic groups and qualitatively better in the control group.
\end{abstract}

Key words: Bone transplantation. Diabetes mellitus. Wound healing.

\section{INTRODUCTION}

The loss of bone tissue from the alveolar ridge, as a cause of pathologies, periodontitis or invasive surgical procedures, may cause structural, functional and esthetic impairments to the dental arch, generating mechanical problems and hindering the installation of prostheses and implants. In an attempt to improve the repair process or even to recover the alveolar anatomy, graft materials and bone substitutes have been used to fill these defects.

Autogenous bone graft has become the material with the highest biological acceptance and tissue compatibility, presenting minimal inflammatory response. However, the clinical success of bone grafts depends on inflammatory events that are controlled by regulatory mechanisms, represented by hormones (insulin, glucocorticoids) and specific tissue factors. Any alteration in those mechanisms, particularly diabetes mellitus, an endocrine alteration, may induce unfavorable reactions in the normal inflammatory process, possibly causing great changes in the revascularization, incorporation, substitution and remodeling grafts stages

The aim of this study was to evaluate the bone repair process in surgical defects prepared in the tibiae of noncompensated diabetic rats and filled with autogenous bone and blood clot.

\section{MATERIALAND METHODS}

Forty male rats (Rattus Norvegicus albinus, Wistar) were randomly assigned to receive an endovenous injection in 
the penile vein of either citrate buffer solution $0.01 \mathrm{M}, \mathrm{pH}$ 4.5 (Group 1 - control; $\mathrm{n}=20$ ) or streptozotocin (SigmaAldrich Corp., St. Louis, MO, USA) dissolved in citrate buffer solution at $35 \mathrm{mg} / \mathrm{kg}$ body weight for experimental induction of diabetes (Group 2 - diabetic; n=20). Five days after induction of diabetes, blood samples were collected from each animal to determine the glycemic levels using an automatic blood glucose monitoring system (Accu-Check ${ }^{\circledR}$; Roche-Diagnostics Corporation, Indianapolis, IN, USA). Seven days after confirmation of hyperglycemia (glycemia $>350 \mathrm{mg} / \mathrm{dL}$ ), the animals were sedated and the anterolateral regions of the tibiae of both posterior limbs were shaved, antisepsis was performed and a longitudinal incision was made in each limb. The tibiae were exposed and two 2-mmdiameter surgical defects were prepared with trephine burs, one in each limb. The defect in the right posterior limb was filled with autogenous particulate bone collected from both surgical defects, whereas the defect in the left posterior limb was filled with blood clot. The animals were euthanized at 10 and 30 postoperative days and the pieces were submitted to routine laboratorial processing and stained with hematoxylin and eosin and Masson's Trichrome for histomorphometric and histologic analyses. The histologic analysis was performed by inflammatory cell count and the histomorphometric analysis was performed with the aid of "Imagelab 2000” image-analysis software. The results were analyzed statistically by Kruskal-Wallis test according to normal curve adherence. Significance level was set at 5\%.

\section{RESULTS}

\section{Histologic Results \\ 10 days}

Group I (Control)

Defect filled with blood clot (CC10): immature bone trabeculae were observed with wide intratrabecular spaces filled with connective tissue with collagen fiber bundles maintaining their organization, large number of fibroblasts and moderate number of inflammatory cells, mainly lymphocytes and macrophages.

Defect filled with Graft (CG10): immature bone trabeculae were observed with wide intratrabecular spaces. Inside the surgical defects there were bone tissue fragments corresponding to the grafts. Close to the newly formed bone tissue margins and the graft fragments, there were numerous osteoblasts characterizing areas with intense bone apposition (Figure 1).

\section{Group II (Diabetic)}

Defect filled with blood clot (DC10): although the bone trabeculae were more numerous, they were visibly thinner compared to those of the control group. Intratrabecular spaces were wide, with well vascularized connective tissue, showing few collagen fiber bundles without organization, several fibroblasts and moderated number of inflammatory cells.

Defect filled with graft (DG10): similarly to the control group, it was possible to observe immature bone trabeculae also presenting wide intratrabecular spaces. There were bone tissue fragments corresponding to the grafts inside the surgical defect. Part of those fragments was devitalized, which was noted by the absence of osteocytes inside the mineralized material. Osteoblasts around the newly formed bone and the graft fragments demonstrated new bone formation activity, though less organized than in the control group (Figure 2).

\section{0 days}

Group I (Control)

Defect filled with blood clot (CC30): The surgical site in all specimens was repaired by well developed bone tissue. There was a greater amount of newly formed bone tissue close to the surgical wound margins, decreasing as it got closer to the central area of the defect (Figure 3).

Defect filled with graft (CG30): It was observed the total closing of the defect by well developed bone. The repaired cortical bone thickness was larger than in the control group filled with blood clot, which is due to the incorporation of bone grafts in the area (Figure 4). There were few intratrabecular spaces, which were filled with collagen fibers, several osteoblasts and few inflammatory cells.

\section{Group II (Diabetic)}

Defect filled with blood clot (DC30): The surgical site was totally repaired by newly formed bone. However, the thickness of the repaired cortical decreased as closer it got from the defect central area, being even thinner than its respective control group. The newly formed bone was thicker at the wound margins, becoming thinner than the respective control group as closer it got to the central area. The bone lamellae were also more disorganized and there were a large number of wide intratrabecular spaces, with several osteoblasts, blood vessels and inflammatory cells (Figure 5).

Defect filled with Graft (DG30): It was observed the total closing of the defect by well formed bone. However, the bone layer was thinner than in the corresponding control group. Bone grafts were incorporated to the newly formed bone by thin bone trabeculae, with wide intratrabecular spaces, several collagen fibers in organization, numerous osteoblasts, blood vessels and few lymphocytes and macrophages. Some specimens presented moderate inflammatory infiltrate, especially those in which it was still possible to visualize the graft resorption process. Regarding the quality, the newly formed bone structure of this group was more immature, as demonstrated by the disorganization of its bone lamellae and osteocytes. Although the bone grafts remained vital, part of the osteocyte lacunas were empty (Figure 6).

\section{Histometric Results}

The amount of newly formed bone is presented in Table 1.

Quantitatively, the results of new bone formation in tibia 


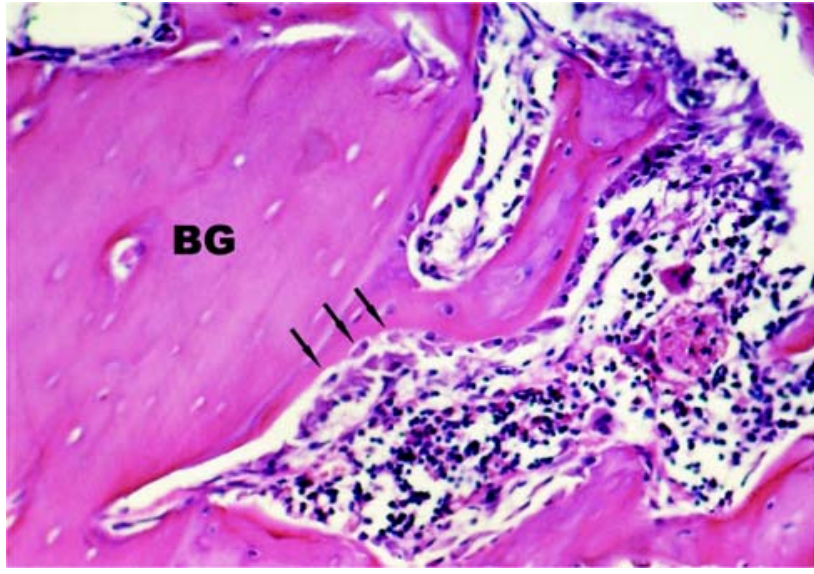

FIGURE 1- Group CG10: Bone fragments corresponding to the grafts (BG) and numerous osteoblasts (arrows) close to the newly formed bone tissue margins and the graft fragments. HE. (Original magnification x160)

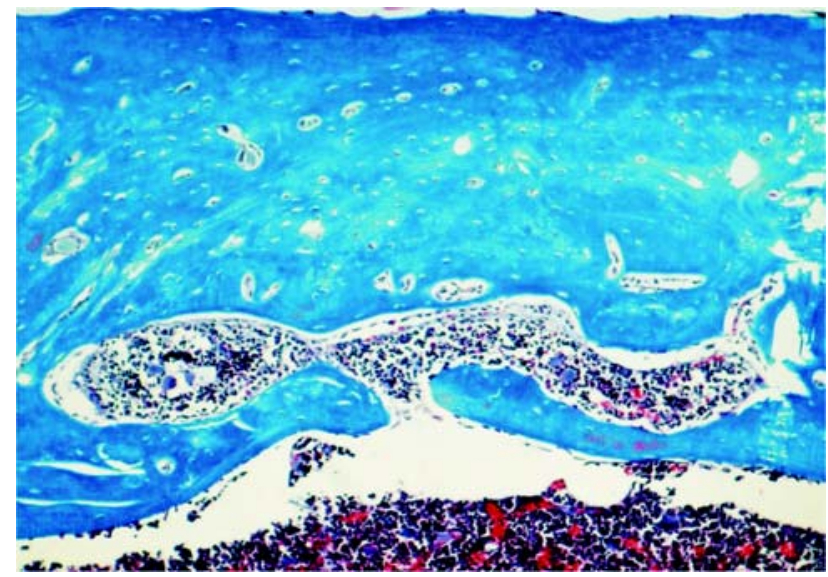

FIGURE 3- Group CC30: Higher amount of newly formed bone closing the surgical site margins and decreasing as closer it got to the defect central area. Masson's Trichrome (Original magnification $\times 63$ )

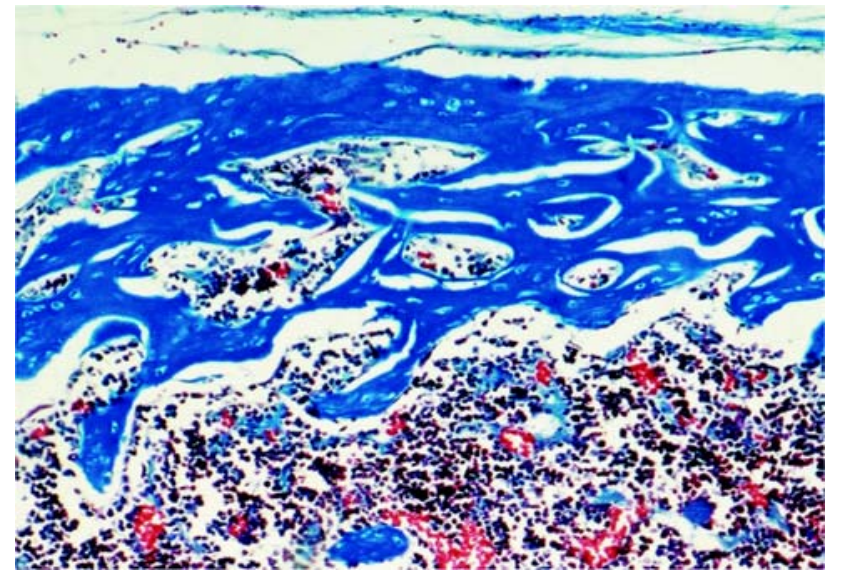

FIGURE 5- Group DC30: Surgical defect repaired by disorganized bone tissue with remnant of wide intratrabecular spaces and cortical thickness decrease at the defect central area. Masson's Trichrome (Original magnification $\times 63$ )

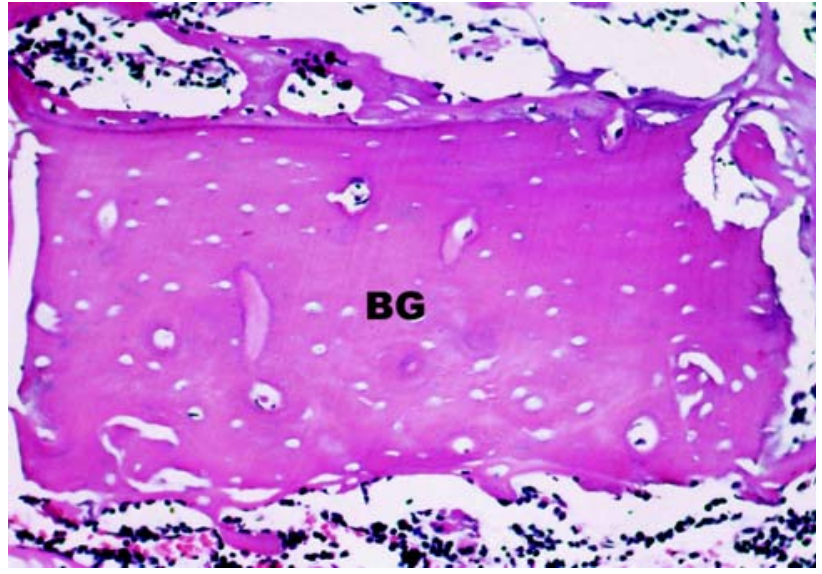

FIGURE 2- Group DG10: Devitalized bone graft (GB) characterized by the absence of osteocytes inside the mineralized material. Osteoblasts around the newly formed bone and the graft, showing new bone formation activity. HE. (Original magnification $\times 160$ )

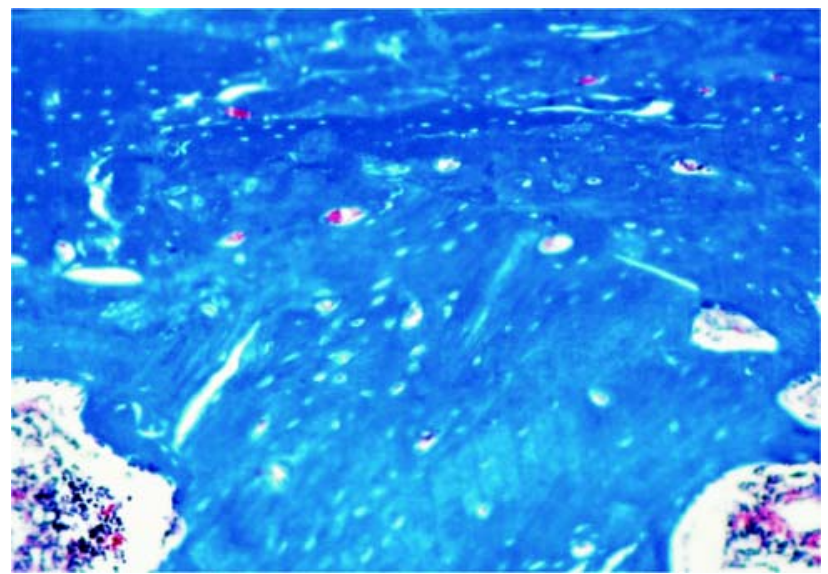

FIGURE 4- Group CG30: Total closing of the defect by well formed bone. Bone graft is totally incorporated to the newly formed bone area. Masson's Trichrome (Original magnification $\times 63$ )

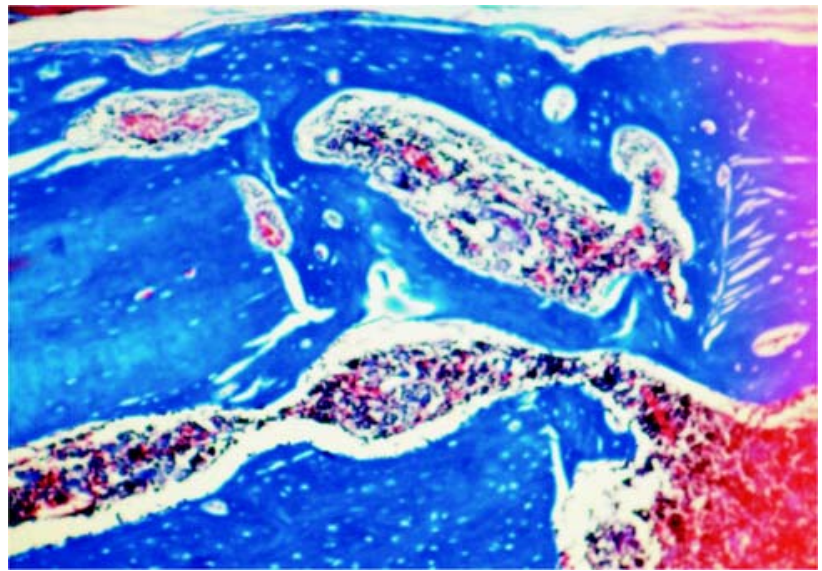

FIGURE 6- Group DG30: Bone grafts incorporated to the newly formed bone by thin bone trabeculae, with wide intratrabecular spaces and disorganized osteocyte lamellae. Masson's Trichrome (Original magnification x63) 
cortical in the grafted surgical defects at the 10 th postoperative day differed significantly $(\mathrm{p}<0.05)$ from those observed in the surgical defects that received the blood clot. In the control group, the percentage of bone area formed in the defects that received graft and blood clot was $52.5 \pm$ $9 \%$ and $42.4 \pm 7 \%$, respectively. At 30 days, although there was a larger newly formed bone area in the grafted defects (75.9 $\pm 11 \%)$ compared to the defects filled with blood clot (71.3 $\pm 7 \%$ ), this difference was not significant ( $p>0.05$ ).

Similar results were observed in the diabetic group at 10 and 30 postoperative days, which presented significantly larger bone area in the grafted defects $(52.0 \pm 7 \%$; $<<0.05$ and $68.9 \pm 12 \%$, respectively; $\mathrm{p}<0.01$ ) compared to the defects filled with blood clot (43.8 $\pm 4 \%$ and $50.7 \pm 13 \%$, respectively).

In the defects that received blood clot, an unexpected inversion of results occurred at the 10-day period (43.8 \pm $4 \%)$, since the diabetic group presented a slightly more accentuated new bone formation than that observed in the control group (42.4 $\pm 7 \%)$, though without statistical significance. However, at the 30-day period, higher bone repair was observed in the control group $(71.3 \pm 7 \%)$ compared to the diabetic group $(50.7 \pm 13 \%)$ (Table 1$)$. Regarding the surgical defects filled with autogenous bone graft, the bone repair in diabetic rats occurred later than in the control animals in both evaluated periods (DG10 $=52.0$ $\pm 7 \%<$ CG10 $52.5 \pm 9 \%$ and DG30 $=68.9 \pm 12<$ CG30 $=75.9 \pm$ 11\%) (Table 1).

TABLE 1- Percentage of newly formed bone per group

\begin{tabular}{lllll}
\hline Group & \multicolumn{2}{c}{$\mathbf{1 0}$ days } & \multicolumn{2}{c}{30 days } \\
Mean (\%) & $\mathbf{n}$ & Mean (\%) \\
\hline CC & 9 & $42.4 \pm 7$ & 10 & $71.3 \pm 7$ \\
CG & 9 & $52.5 \pm 9$ & 10 & $75.9 \pm 11$ \\
DC & 10 & $43.8 \pm 4$ & 10 & $50.7 \pm 13$ \\
DG & 10 & $52.0 \pm 7$ & 10 & $68.9 \pm 12$ \\
\hline
\end{tabular}

\section{DISCUSSION}

Diabetes mellitus is a well known disease that weakens the patient's general metabolism ${ }^{7}$ and changes the cellular and humoral immunity ${ }^{9,16,17}$, increasing the risk of infections when the organism is submitted to surgical procedures ${ }^{1,14,15}$. Moreover, the delay in cell proliferation ${ }^{12}$, the decrease of collagen metabolism ${ }^{5}$ and all other granulation tissue components, such as glycoproteins and mucopolysaccharides ${ }^{4,6}$, are direct consequences of the disease that severely affects the tissue repair process.

In the experimental model used in this study, the animals developed streptozotocin-induced diabetes. This substance produces irreversible effects on the pancreas and generates, after induction of diabetes, irreversible organic metabolic conditions similar to those observed in humans. The diabetic status occurs in the first hour after drug administration, but the clinical signs of this new systemic condition appear only $24 \mathrm{~h}$ after streptozotocin injection ${ }^{3}$. Determination of the glycemic levels and surgical procedures were performed 5 and 7 days after induction of diabetes, respectively, in order to allow adaptation of the animals to the new metabolic condition and thus minimize the possibility of variations in their physiological behavior.

The bone repair process is particularly affected in diabetic individuals because the hyperglycemic status leads to an increase of bone resorption and a decrease of bone turnover $^{8}$. In addition, there is a decrease in osteoblastic differentiation ${ }^{10}$ and a delay in the revascularization process $^{2,11}$, which, according to Santana, et al. ${ }^{13}$ reduces by approximately $60 \%$ the bone repair ability compared to a healthy organism.

Comparing the sub-groups "blood clot" and "autogenous bone graft" within the control and diabetic group, the treatment of bone wounds with autografts promoted significantly better results than filling of the defects with blood clot at both periods (10 and 30 days).

Although similar results were found between control and diabetic groups after histometric analysis of the bone area, the results were qualitatively very distinct. The presence of intratrabecular spaces with connective tissue still in organization was the main characteristic of the diabetic wounds at 10 days. The inflammatory infiltrate also seemed more intense when compared to the control group in the initial repair periods. Collagen fiber deposition is less intense and less organized in the diabetic organism, especially in the earlier repair periods.

Bone graft vitality also seemed to be reduced in the diabetic group because, in addition to having thinner and disorganized trabeculae, there was an intense osteoclastic activity, especially in the inner margins of the bone grafts. Empty osteocyte lacunas were found in some experimental diabetic animals (Figure 2).

During the analysis of the groups that received only blood clot in the defects, it was verified an unexpected inversion of the results from the 10-day period, as the new bone formation that occurred in the diabetic group was slightly more accentuated when compared to the control group. However, it is important to emphasize that the quality of the newly formed bone in the diabetic-blood clot group at 10 days, as well as the bone constituents present in the intratrabecular spaces, such as inflammatory infiltrate, newly formed vessels and collagen fibers, showed slightly worse characteristics. At the 30-day period, the results followed expected patterns, with a prevalence of bone formation in the control group when compared to the diabetic group.

Although complete repair of the surgical defects by newly formed bone tissue at 30 days was observed in all specimens, the presence of intratrabecular spaces was more persistent in the diabetic group, with a larger number of blood vessels and discreetly greater inflammatory infiltrate, which resulted in a more accentuated qualitative impairment of the repair 
process.

Incorporation of bone fragments was also observed in both groups and periods. However, in spite of the smaller inflammatory infiltrate and the larger number of osteoblasts and collagen fibers in the intratrabecular spaces of the control group, the diabetic organism response to bone grafts was considered as very satisfactory, given that the repair process in diabetics is recognizably impaired ${ }^{2}$.

Although similarities were found between the groups at the earlier stages of the alveolar repair, it is likely that great part of the changes in tissue repair in the diabetic animals are due to the delays in the proliferative stage of the repair process $^{2,12}$. It is likely that such alterations led to a qualitative bone repair delay, although the greatest discrepancies between the groups were more evident at 30 postoperative days.

\section{CONCLUSIONS}

Based on the results of the present study, it may be concluded that: 1 . The bone repair of surgical defects filled with bone grafts occurred earlier than that of surgical defects filled with blood cot in both control and diabetic groups; 2. Regarding surgical defects filled with blood clot, the repair process in the control group was qualitatively better than the repair in diabetic group in both evaluated periods, with significant difference at 30 days period; 3 . Regarding the surgical defects filled with bone grafts, the control group showed better bone repair than the diabetic group, though without statistical significance.

\section{ACKNOWLEDGEMENTS}

This study was supported by the São Paulo State Research Foundation (FAPESP), Brazil (grant 04/13990-0).
7- Graves DT, Liu R, Oates TW. Diabetes-enhanced inflammation and apoptosis: impact on periodontal pathosis. Periodontol 2000. 2007;45:128-37.

8- Krakauer JC, McKenna MJ, Buderer NF, Rao DS, Whitehouse FW, Parfitt AM. Bone loss and bone turnover in diabetes. Diabetes. 1995;44(7):775-82.

9- Liu R, Desta T, He H, Graves D. Diabetes alters the response to bacteria by enhancing fibroblast apoptosis. Endocrinology. 2004;145:2997-3003.

10- Lu H, Kraut D, Gerstenfeld L, Graves D. Diabetes interferes with the bone formation by affecting the expression of transcription factors that regulate osteoblast differentiation. Endocrinology. 2003;144:346-52.

11- Maitra A, Abbas. A The endocrine system. In: Kumar V, Fausto N, Abbas A. Robbins \& Cotran pathologic basis of diseases. Philadelphia: Sauders; 2004. p.1543-60.

12- Maruyama K, Asai J, Li M, Thorne T, Losordo DW, D‘Amore PA. Decreased macrophage number and activation lead to reduced lymphatic vessel formation and contribute to impaired diabetic would healing. Am J Pathol. 2007;170:1178-91.

13- Santana RB, Xu L, Chase HB, Amar S, Graves DT, Trackman PC. A role for advanced glycation end products in diminished bone healing in type 1 diabetes. Diabetes. 2003;52:1502-10.

14- Senel FC, Jessen GS, Melo MD, Obeid G. Infection following treatment of mandible fractures: the role of immunosuppression and polysubstance abuse. Oral Surg Oral Med Oral Pathol Oral Radiol Endod. 2007;103(1):38-42.

15- Sykes LM, Sukha A. Potential risk of serious oral infections in the diabetic patient: a clinical report. J Prosthet Dent. 2001;86(6):569-73.

16- Walrand S, Guillet C, Boirie Y, Vasson MP. In vivo evidences that insulin regulates human polymorphonuclear neutrophil functions. J Leukoc Biol. 2004;76(6):1104-10.

17- Walrand S, Guillet C, Boirie Y, Vasson MP. Insulin differentially regulates monocyte and polymorphonuclear neutrophil functions in healthy young and elderly humans. J Clin Endocrinol Metab. 2006;91(7):2738-48.

\section{REFERENCES}

1- Abdulwassie H, Dhanrajani PJ. Diabetes mellitus and dental implants: a clinical study. Implant Dent. 2002;11(1):83-6.

2- Brem H, Tomic-Canic M. Cellular and molecular basis of wound healing in diabetes. J Clin Invest. 2007;117(5):1219-22.

3- Covington DS, Xue H, Pizzini R, Lally KP, Andrassy RJ. Streptozotocin and alloxan are comparable agents in the diabetic model of impaired wound healing. Diabetes Res. 1993;23(2):47-53.

4- Desmouliere A, Redard M, Darby I, Gabbiani G. Apoptosis mediates the decrease in cellularity during the transition between granulation tissue and scar. Am J Pathol. 1995;146:56-66.

5- Falanga, V. Wound healing and its impairment in the diabetic foot. Lancet. 2005;366(9498):1736-43.

6- Galkowska H, Wojewodzka U, Olszewski WL. Chemokines, cytokines, and growth factors in keratinocytes and dermal endothelial cells in the margin of chronic diabetic foot ulcers. Wound Repair Regen. 2006;14:558-65. 